\title{
Methodology
}

\section{Multiple attribute scenarios, bounded probabi- lities, and threats of nuclear theft}

\author{
Craig W. Kirkwood and Stephen M. Pollock
}

A method is presented for developing descriptions of future scenarios and using expert judgment to assess bounds on the probabilities of these scenarios. Multiple attributes are used to describe the important features of the scenarios, and the scenarios are defined as collections of different possible levels of the attributes. Experts assess either numerical values or bounds on various unconditional and conditional probabilities for different attribute levels. These are used to establish constraints for a series of linear programs which are solved to determine the highest and lowest possible probabilities for each scenario. An application is presented to the assessment of potential threats against nuclear material safeguards systems.

\section{Keywords: planning; scenarios; nuclear safeguards}

Systematic planning for the future usually involves forecasting relevant future conditions. Significant features of the future are often uncertain, and it is desirable to account explicitly for these uncertainties in planning. The use of scenarios-internally consistent descriptions of possible future conditions and events-has expanded over the past 15 years. With this approach, several different scenarios are constructed which cover the range of possible future conditions, and the probability of each

Craig W. Kirkwood is with Woodward-Clyde Consultants, Three Embarcadero Center, Suite 700, San Francisco, C:A 94111, USA; and Stephen M. Pollock is at the Department of Industrial and Operations Engineering, The University of Michigan, Anu Arbor, MI 48109, USA. This work was supported in part by the University of California Lawrence Livermore Laboratory under Purchase Order No. 2005909 with WoodwardClyde Consultants, as part of work on Contract No. W7405ENG.48 with the US Energy Research and Development Administration. future scenario is estimated. This information is then used in planning and decision making.

One difficulty with this method is that historical information may be only indirectly relevant to estimation of probabilities for future scenarios, and it may thus be necessary to use some other method to determine these probabilities. This paper presents a method for using expert judgment to do this which overcomes certain limitations of past approaches. We also present an application of the method.

\section{Background}

The use of scenarios in planning has received increasing attention over the past 15 years. ${ }^{1}$ In this section we review cross-impact analysis (which is closely related to the work presented here) and discuss limitations in previous methods for implementing this approach. The 
basic features of cross-impact analysis are illustrated by SMIC 74,2 in which the following steps are carried out:

1. Events $e_{1}, e_{2}, \ldots, e_{\mathrm{n}}$ are constructed, each being considered relevant to the scenarios of interest.

2. The unconditional probability $p\left(e_{i}\right)$ of each event occurring is assessed from experts.

3. The conditional probabilities $p\left(e_{i} e_{j}\right)$ and $p\left(e_{i} e j\right)$ are also assessed, where $e_{j}$ is the complement of $e_{j}$.

4. The assessed information is corrected so that for the final results

$$
\begin{aligned}
& 0 \leqslant p\left(e_{i}\right) \leqslant 1, \\
& p\left(e_{i} \mid e_{j}\right) p\left(e_{j}\right)=p\left(e_{j} \mid e_{i}\right) p\left(e_{i}\right), \text { and } \\
& p\left(e_{i} \mid e_{j}\right) p\left(e_{j}\right)+p\left(e_{i} \mid e_{j}\right)=p\left(e_{i}\right) .
\end{aligned}
$$

The correction procedure involves minimizing a quadratic difference function subject to meeting the constraints given in step 4 above. Various authors have pointed out limitations in the original SMIC 74 procedures and have suggested modifications.3 In particular, Mitchell and Tydeman ${ }^{+}$have demonstrated the subtleties involved in eliciting conditional probabilities when an implicit time dependence between events of interest can influence the experts' probability assessments. Mitchell and Tydeman emphasize the need to carefully consider the order of temporally sequenced events if elicited probabilities are to be meaningful. These authors address inconsistencies in assessed probabilities by hypothesizing that there is a true or consistent value for each inconsistent probability assessed by the experts, and using mathematical programming methods to estimate a consistent set of probabilities that is as close as possible to the experts' assessments.

Sarin has proposed a somewhat different approach where probability information on events is collected sequentially from experts. ${ }^{5} \mathrm{~A}$ computer program is used to check the consistency of the elicited infornation with the axioms of probability. If the information is consistent, then bounds on feasible values of the joint and conditional probabilities $p\left(e_{i}, e_{j}\right)$ and $p\left(e_{i} e_{j}\right)$ are calculated and presented to the experts. The experts continue to provide additional information until the calculated bounds are considered tight enough.

These approaches to determining scenario probabilities have two limitations. First, the number of scenarios that must be considered can quickly become excessive; 10 binary events will give $2^{10}$ $=1024$ different possible scenarins. Mitchell, Tydeman and Curnow show that a large fraction of these can each have a non-negligible probability. Not only may it be difficult to analyse all these scenarios, it may not be necessary to consider this much detail for the planning problem of interest.

A second limitation is that they deal with binary events which either do, or do not, occur; in many planning problems, it is more natural to consider attributes (factors) which can take on more than two different levels. For example, there is no natural way to describe the price of oil simply in terms of events either occurring or not.

In the next section we discuss a new approach which addresses these two limitations.

\section{Approach}

Our basic approach can be illustrated by a simplified example. Suppose an electric utility is deciding whether to plan construction of either a coal- or an oil-fired steam turbine power plant to supply its customers from 1995 to 2035 . To assist in its planning, the utility wishes to consider possible scenarios for conditions in that time period. Two factors, relative cost $\left(e_{1}\right)$ and regulations $\left(e_{2}\right)$, are considered relevant. These factors can take on the following levels:

$$
e_{1}=\left\{\begin{array}{l}
1: \text { coal is substantially } \\
\text { cheaper than oil, } \\
2: \text { the two fuels have } \\
\text { sinilar custs, and } \\
3: \text { oil is substantially } \\
\text { cheaper than coal. }
\end{array}\right.
$$




$$
e_{2}=\left\{\begin{array}{l}
1: \text { regulations forbid use } \\
\text { of oil, and } \\
2: \text { regulations permit use } \\
\text { of oil. }
\end{array}\right.
$$

There are six scenarios, or different possible combinations of these factors. However, let us assume that for planning purposes the utility need only consider the following four scenarios:

$S_{i}$ : regulations forbid use of oil;

$S_{2}$ : regulations permit use of oil, and oil is substantially cheaper than coal;

$S_{3}$ : regulations permit use of oil, and the two fuels have similar costs; and

$S_{4}: \quad$ regulations permit use of oil, and coal is substantially cheaper than oil.

While experts might provide probability information in terms of $e_{1}$ and $e_{2}$, it is only necessary to obtain probabilities for $S_{1}, S_{2}, S_{3}$, and $S_{4}$. If joint probabilities are available over $e_{1}$ and $e_{2}$, then it is straightforward to obtain probabilities for the scenarios.

In this illustration, the saving obtained by defining the four scenarios is not great: there are only two more possible combinations of $e_{1}$ and $e_{2}$ than scenarios. In the application discussed below, there are eight attributes with a total of 512 different possible conbinations of levels-but only 19 scenarios are needed to adequately describe possible future conditions for planning purposes. Since it will be necessary only to obtain the probabilities for these 19 scenarios, considerably less elicitation will be needed than if probabilities were to be obtained for all 512 possible combinations of attribute levels.

Our approach to determining scenario probabilities, based on the ideas just illustrated, has the following steps:

1. Determine a set of discrete valued attributes $e_{1}, e_{2}, \ldots, e_{n}$ which cover all concerns that are relevant for the planning problem of interest.
2. Determine the set of relevant scenarios for the planning problem. This set will be collectively exhaustive and mutually exclusive over the possible combinations of $e_{1}, e_{2}, \ldots$, $e_{n}$.

3. Assess, with an expert, information about unconditional probabilities, conditional probabilities and joint probabilities over the attributes $e_{1}$, $e_{2}, \ldots, e_{n}$ which he or she can provide.

4. Determine whether the assessed probabilities are consistent with the axioms of probability theory. If not, resolve the inconsistencies with the expert; if so, determine upper and lower bounds on the probability of each scenario that are consistent with the assessed information.

5. If these bounds are sufficiently tight for planning purposes, stop; otherwise, assess additional information and redetermine the bounds.

Note that step 3 does not require assessments for prespecified sets of unconditional, conditional, or joint probabilities. The respondent need only assess those probabilities with which he or she is comfortable.

For notational convenience, let $T$ represent the set of all possible combinations of $e_{1}, e_{2}, \ldots, e_{n}$, let $t$ represent a specific element in $T$, and $p_{\mathrm{t}}$ represent the probability of $t$. Now consider an elicitation procedure that allows an expert to provide numerical judgmental values, or upper or lower bounds, for:

i. unconditional probabilities for any attribute, or

ii. conditional probabilities for any single attribute, given information about the other attributes.

In addition, let the procedure allow:

iii. comparison of the magnitude of the probabilities of any two specific elements $t$ and $u$ in $T$.

The representation of this information in terms of $p_{t}$ is given as follows:

Unconditional probabilities. Let $E_{i j}$ 
represent all elements in $T$ such that $e_{i}=$ $j$. Then an assessed unconditional probability $\operatorname{Prob}\left\{e_{i}=j\right\}=p$, as discussed in itcm $i$ above, imposes the constraint:

$$
\begin{aligned}
& \sum P_{t}=p . \\
& t \varepsilon E_{i j}
\end{aligned}
$$

An assessed upper bound $\operatorname{Prob}\left\{e_{i}=j\right\} \leqslant$ $p$ imposes

$$
\begin{aligned}
& \sum P_{i} \leqslant p, \\
& \imath \varepsilon E_{i j}
\end{aligned}
$$

while a lower bound $\operatorname{Prob}\left\{e_{i}=j\right\} \geqslant p$ leads to

$$
\begin{aligned}
& \sum P_{i} \geqslant p . \\
& \iota \varepsilon E_{i j}
\end{aligned}
$$

Conditional Probabilities. Let $\bar{E}_{i}$ represents a specified set of levels for all attributes except the $i^{\text {th }}$. The conditional probability that the $i^{\text {th }}$ attribute equals $e_{i}$, given the other attributes are in $\vec{E}_{i}$, will be denoted $P\left(e_{i} \bar{E}_{i}\right)$; further, let $\left(e_{i}, \bar{E}_{i}\right)$ be the set of $t$ such that the $i$ thattribute equals $e_{i}$ and the other attributes are in $\bar{E}_{i}$. Then an assessed conditional probability $p$, as discussed in item ii above, imposes one of the following constraints:

$$
\begin{aligned}
& P\left(e_{i} \mid \bar{E}_{i}\right) \leqslant p \\
& P\left(e_{i} \mid \bar{E}_{i}\right)=p \\
& P\left(e_{i} \mid \bar{E}_{i}\right) \geqslant p
\end{aligned}
$$

Here Equation (2a) applies when an upper bound is assessed, (2b) when a specific numerical value is assessed, and (2c) when a lower bound is assessed.

The expressions in Equation (2) can be rewritten in terms of $p_{t}$ by applying standard conditional and total probability definitions:

$$
\underset{t \varepsilon\left(e_{i}, \bar{E}_{i}\right)}{\sum p_{l} p^{\prime} \sum P_{t} P_{\mathrm{t}}} \leqslant 0,
$$

or it is equal to zero, or it is greater than zero.

Magnitude of two elements. The comparison of probabilities for two specific elements in $T$, as discussed in item iii above, imposes one of the following constraints: $P_{t}$ greater than, equal to, or less than $P_{u}$.

Bounds on scenario probabilities. Let $S_{1}$, $S_{2}, \ldots, S_{m}$ represent the set of scenarios used, where this set is collectively exhaustive and mutually exclusive over $T$. Then the upper bound on the probability, $P\left(S_{i}\right)$ of $S_{i}$ occurring, where

$$
P\left(S_{\mathrm{i}}\right)=\sum_{t \varepsilon S_{i}},
$$

can be found by solving the linear program

$$
\underset{\left[p_{t}, t \varepsilon T\right]}{\operatorname{maximize}} \sum_{t \varepsilon S_{i}} P_{t}
$$

subject to

$$
\begin{aligned}
& \begin{array}{l}
\text { assessed constraints of type } \\
(1),(3), \text { and }(4)\}
\end{array} \\
& \sum p_{t}=1 \\
& t \varepsilon T
\end{aligned}
$$

and

$$
p_{t} \geqslant 0, t \varepsilon T
$$

Similarly, the lower bound on $P\left(S_{i}\right)$ can be found by solving the linear program

$$
\underset{\left\lceil p_{t}, t \varepsilon T\right] \quad \operatorname{minimize}_{i}}{\sum} P_{t}
$$

subject to Equations (5b, c, d).

Thus, to find the bounds on the probabilities for each of the $m$ scenarios, it is necessary to solve $2 m$ linear programs. With current linear programming computer packages this is inexpensive, even for large problems. In the event there is no feasible set of $p_{t}$, the phase I portion of the simplex solution algorithm will automatically identify this.

Although the theoretical formulation presentcd above is straightforward, keeping track of the $E_{i j}, p, \bar{E}_{i}, p$, and $S_{i}$ in an actual application requires 
developing special procedures. In the next section, we discuss an application of the approach and procedures for data handling. Similar procedures should be applicable to a variety of planning problems.

\section{Application}

The application discussed below was carried out as part of a study to assist the University of California Lawrence Livermore Laboratory in the development of methods to analyse and evaluate Nuclear Material Safeguards Systems (NMSS). Our study concentrated on developing a methodology to assist experts in describing in quantitative form their judgments about scenarios characterizing potential threats to steal material from a NMS system. (Such a system is a combination of guards, alarms, procedures, and other steps taken at a facility handling nuclear materials to keep the material safe from hostile action.) The ultimate purpose for developing these scenarios, and their associated probabilities, was to aid design work to improve the performance of NMS systems.

To develop scenarios and associated probabilities, we followed the five-step process discussed in the last section. Our main purpose was development of methodology, and the work presented below does not represent a final characterization of adversaries.

During Step 1 of our process, eight attributes were determined to characterize potential adversaries (see Table 1), and the scales also shown in the Table were constructed to describe possible levels of each attribute that an adversary might possess. Standard decision analysis methods were used in determining the attributes and scales. ${ }^{7}$ Care was taken to assure that the set of scales was complete, nonredundant, operational, and of minimum size.

With the attribute levels shown in Table 1 , there are $4 \times 2^{7}=512$ different possible scenarios characterizing adversaries. However, examination of the various different possible combinations of attribute levels showed that many of these would pose similar threats to a NMS system. Since the planning problem of interest was to assist in improving such systems, there was no need to distinguish between different adversaries who posed similar threats.

A detailed consideration of the various possible threat scenarios, carried out during Step 2 of the process discussed in the last section, identified the 19 distinct threat scenarios described in Table 2.

These scenarios are more explicitly described as collections of the 512 different types of adversaries defined by the various possible combinations of attribute levels in Table 1. To represent conveniently the scenario definitions, a straightforward coding procedure was used. For example, the code $(3,1,1,2,1,1,2,1)$ represents an adversary whose motivation is extortion (level 3 of the first attribute on Table 1), and who has high levels of NMS system information and technical information, low consequence information, high levels of processing rapability and general resources, and is risk-avoiding for himself but risk-seeking for others. If any cntry is zero, there is no restriction on that particular attribute, and so the code refers to a collection of more than one of the 512 possible adversaries. Thus, the code $(3,0,1,0,0,0,0,0)$ represents all adversary descriptions where the motivation is extortion and there is a high level of technical information.

Each of the 19 threat scenarios in Table 2 was represented in terms of this code. Thus, for example, scenario 1 (Uninformed Outsider) had code $(1,2,2,0,0,0,0,0), \quad$ scenario 8 (Embezzling Executive) had code $(2,1,1,0,0,2,0,0)$ and scenario 14 (Outside Expert) had code $(3,2,1,0,0,2,0,0)$. A description of the codes for all scenarios is given by 
Attribute

1 Motivation

2 NMS system information

3 Technical information

4 Consequence information

5 Processing capability

6 General resources

7 Self-risk attitude

8 Other's-risk attitude

\section{Area of concem \\ Reason a theft is attempted}

Level of knowledge about the operation of Nuclear Material Safeguards Systems

Level of knowledge about nuclear materials, their handling, and their processing

Level of knowledge about possible legal, financial, social, and health consequences of a theft attempt

Equipment and expertise available to process stolen nuclear material

Willingness of adversary to risk lives and welfare of those not in the adversary group

Resources, such as equipment and number of personnel, available to aid in theft attempt

Willingness of adversary to risk his own life and welfare

\section{Levels}

1 Symbolic: eg game playing prank, crazy, personal revenge, for principle, publicity for a cause, cover-up of material unaccount for

2 Money: To make money by sale of the material or ransom for its safe retum

3 Extortion: Theft for nonmonetary gain (eg political gain, job restitution, prisoner release)

4 Weapon: Theft to make a weapon for eventual use (eg explosive device or radiation dispersal weapon)

1 High: Considerable knowledge of and control over at least one aspect of the Nuclear Material Safeguards System

2 Low: Has only the knowledge available to someone outside the NMS system

1 High: Has significant knowledge about nuclear science, computer technology, electronics, and related areas

2 Low: Does not have significant knowledge about these areas

1 High: Fullunderstanding of the possible legal, financial, social, and health consequences of the theft attempt

2 Low: Incomplete understanding of the possible consequences

1 High: Has the equipment and expertise to process stolen material, either to make a weapon or to enrich or alter itfor easier storage or sale

2 Low: Does not have such capabilities

1 High: a group of people with some experience in theft and criminal activity

2 Low: a group or individual inexperienced in theft and criminal activity

1 Seeking: Adversary will take chances which threaten his own life or welfare

2 Avoiding: Adversary will not accept significant probability of loss of life or capture.

1 Seeking: Adversary is willing to risk lives and welfare of those not in adversary group

2 Avoiding: Adversary unwilling to do this
Kirkwood and Pollock."

Assessing probabilities. A detailed questionnaire was prepared to assess judgments from experts concerning the probabilities that a potential adversary would have the various levels of attributes shown in Table 1. In particular, unconditional probability distributions were assessed over several of the attri- butes as well as conditional probability distributions over these same attributes, given that the level of one other attribute was specified.

The questionnaire furnished general background information on other theft situations that are analogous in some way to theft from a Nuclear Material Safeguards System. The questionnaire 
TABLE 2. ADVERSARY THREAT SCENARIOS

\section{Scenario titie \\ 1 Uninformed outsider \\ 2 Principled outsider \\ 3a Disgruntled employee \\ 3b Disgruntled employees}

4 Principled executive

5a Competent outsider

$5 b$ Competent outsider group

6a Opportunistic employee

6b Opportunistic employees

7 High-level embezzling group

8 Embezzling executive

9 Terrorist group or foreign national group

10 Terrorist individual

11 Insider terrorist group

12 Insider terrorist individual

13 Outsider extortionist group

14 Outside expert

15 Extorting employee

16 Extorting executive

\section{Characteristics}

Theft for symbolic reasons, prankster, crazy, for principle, but not planned; very little Nuclear Material Safeguards System (NMSS) knowledge, technically unsophisticated

Theft for symbolic reasons (ie game-playing, for principle); little or no NMSS knowledge, technically sophisticated; could have considerable diversion resounces, but no inside contacts Symbolic theft (eg labour unrest, revenge on the system, material-unaccounted-for coverup); NMSS low-level insider, such as guard, minor technician, maintenance worker, etc; little technical knowledge

Symbolic theft (eg for principle, revenge, coverup of present or future material-unaccounted-for); high level NMSS position and contacts (executive administrator, etc); likely to understand consequences of theft; high technical knowledge, possibly a group

Theft for monetary gain, (eg future sale); little or no NMSS information; technically sophisticated; has diversion resources

Crime of opportunity, for money; knows one area of NMSS (eg guard, loading dock worker, etc); little technical sophistication

Possibly long-term diversion for profit; well-informed insiders; technically sophisticated; have NMSS contacts, control, and equipment available

Crime of opportunity, possibly long-term diversion; wellinformed insider; technically sophisticated

Theft to build weapon; little or no NMSS information; high technical and processing capability; sophisticated ams and adequate manpower (likely to mount a direct frontal attack) Theft to bulld weapon; little or no NMSS information; technically sophisticated and capable of processing material; few theft resources (likely to attack fringe of system such as material being transported)

Theft to build weapon; well-informed insiders; technically sophisticated with processing capability; have NMSS contacts, control, and equipment available (may be a large group with theft equipment)

Theft to build weapon; well-informed insider; technically sophisticated with processing capability; little NMSS control and few contacts (small group or individual)

Theft for extortion; see $5 \mathrm{~b}$ for other characteristics

Theft for extortion; see 5 a for other characteristics

Theft for extortion, see $6 a, 6 b$ for other characteristics

Theft for extortion; see 8 for other characteristics was self-explanatory, but not designed to be self-administered. The experts were questioned to determine the reasons for their answers and to reduce the chances of the various usual assessment errors in judgmental probabilities."

Determining scenario probability bounds. The two sets of optimization problems (5) and (6) were solved to obtain bounds on the probabilities for the 19 threat scenarios in Table 2 , given the information assessed from the experts. Solution of these linear programs is conceptually straightforward: there are numerous programs available that will solve linear programs larger than these. However, manually entering the data would be very tedious since there were 512 different $p t$ 's and several dozen constraints. 
TABLE 3. BOUNDS ON SCENARIO PROBABILITIES

\begin{tabular}{|c|c|c|c|c|c|c|}
\hline \multirow{2}{*}{$\begin{array}{l}\text { Scenario } \\
\text { number }\end{array}$} & \multicolumn{2}{|c|}{ Expert Aa } & \multicolumn{2}{|c|}{ Expert Ba } & \multicolumn{2}{|c|}{ Expert cb } \\
\hline & $\operatorname{Max}$ & Min & Max & Min & $\operatorname{Max}$ & Min \\
\hline 1 & 0.0526 & 0 & 0.3333 & 0 & 0.01515 & 0 \\
\hline 2 & 0.0526 & 0 & 0.3333 & 0 & 0.01515 & 0 \\
\hline $3 a$ & 0.0526 & 0 & 0.3333 & 0 & 0.04167 & 0 \\
\hline $3 b$ & 0.0526 & 0 & 0.3333 & 0 & 0.04167 & 0 \\
\hline 4 & 0.0526 & 0 & 0.4608 & 0 & 0.1667 & 0.10987 \\
\hline $5 a$ & 0.3 & 0 & 0.0588 & 0 & 0.01515 & 0 \\
\hline $5 b$ & 0.5 & 0 & 0.0588 & 0 & 0.04545 & 0 \\
\hline $6 a$ & 0.3 & 0 & 0.0588 & 0 & 0.01515 & 0 \\
\hline $6 b$ & $0-3$ & 0 & 0.0588 & 0 & 0.125 & 0.02652 \\
\hline 7 & 0.2526 & 0 & 0.0588 & 0 & 0.10987 & 0 \\
\hline 8 & 0.2526 & 0 & 0.0588 & 0 & 0.01515 & 0 \\
\hline 9 & 0.1579 & 0 & 0.0589 & 0 & 0.0303 & 0 \\
\hline 10 & 0.1579 & 0 & 0.0589 & 0 & 0.0303 & 0 \\
\hline 11 & 0.1579 & 0 & 0.0589 & 0 & 0.1666 & 0.06056 \\
\hline 12 & 0.1579 & 0 & 0.0589 & 0 & 0.07575 & 0 \\
\hline 13 & 0.2632 & 0 & 0.2941 & 0 & 0.07575 & 0 \\
\hline 14 & 0.2632 & 0 & 0.2941 & 0 & 0.04545 & 0 \\
\hline 15 & 0.2632 & 0 & 0.2941 & 0 & 0.20833 & 0.12500 \\
\hline 16 & 0.2632 & 0 & 0.2941 & 0 & 0.32955 & 0.21593 \\
\hline
\end{tabular}

Notes:a: using only unconditional probability responses; $b$ : using unconditional probability responses and some conditional probability responses.

To avoid this, a preprocessor program was written to take information about assessed probabilities and set up the appropriate constraint equations for the linear programming solution package. The entire computer procedure to take clicited data, to set up input files for the linear programming solution package, and to run this to obtain bounds on scenario probabilities, was implemented as an interactive program on the Michigan Terminal System at the University of Michigan. The linear program solution package MPS, which is part of the IBM Mathematical Programming System, was utilized. Although the program we developed is straightforward to use, the underlying programs are fairly complex. It is necessary to handle and sort a number of large data files which are either created by the preprocessor or permanently sorted for use by the program.

In order to simplify program use, data was entered using a coding scheme similar to that described earlier. For example, 10000000G0.3 means "the probability that Motivation is Symbolic is greater than $0.3 . " \quad$ Similarly 20000000L30000000 means "the probability that Motivation is Money is less than the probability that Motivation is Extortion." Conditional probabilities were entered using a slight variation in this scheme. For example, $\mathrm{P} 6=\mathrm{IGl}=4 \mathrm{G} 0.7$ means that "the probability that attribute 6 (General Resources) is High, given that attribute 1 (Motivation) is Weapon, is greater than 0.7.'

The preprocessor program recognizes the meaning of these codes and sets up the appropriate constraint equations for the linear programs. The details of the program are presented in Kirkwood and Pollock."

\section{Results of the application}

The probability assessments discussed above were carried out for three experts. All three experts found it possible to give meaningful answers to both the unconditional and conditional probability questions. When attempts werc made to obtain bounds for the scenario probabilities based on the data for each expert, 
it turned out there was no set of $p_{t}$ obeying the axioms of probability theory that matched the elicited data. For two of the experts, the inconsistency remained as long as any of the conditional probability data were used, while with the third, some (but not all) of the conditional data could be utilized and consistency retained. The bounds that result when the inconsistent conditional probability assessments are omitted are shown in Table 3 where the three experts are designated $\mathrm{A}, \mathrm{B}$ and $\mathrm{C}$.

This appliction shows that the methodology in this paper is a viable way of analysing scenarios described by attributes with multiple levels. However, it is difficult to assess consistent probability information in these complex situations.

\section{References}

1. J. Gershuny, "The choice of scenarios", Futures, 1976, 8, pages 496-508; C.A.R. MacNulty, "Scenario development for corporate planning", Futures, 1977, 9, pages 128-138; K. Nair and R.K. Sarin, "Generating future scenarios--their use in strategic planning", Long Range Planning, June 1979, 23, (3), pages 57-61.

2. J.C. Duperrin and M. Godet, "SMIC: 74-A method for constructing and ranking scenarios", Futures, 1975, 7. pages $302-312$.

3. M. Godet, "SMIC 74-A reply from the authors", Futures, 1976, 8, pages 336340; P. Kelly, "Further comments on cross-impact analysis", Futures, 1976, 8 , pages 341-345; R.B. Mitchell and J. Tydeman, "A note on SMIC: 74", Futures, 1976, 8, pages 64-67; R.B. Mitchell and J. Tydeman, "A further comment on SMIC 74", Futures, 1976, 8, pages 340-34l; R.B. Mitchell, J. Tydeman, and R. Curnow, "Scenario generation: limitations and developments in cross-impact analysis", Futures, 1977, 9. pges 205-215.

4. R.B. Mitchell and J. Tydeman, "Subjective conditional probability modelling"., Technological Forecasting and Social Change, 1978, 11, pages 133-152; R.B. Mitchell, "Subjective conditional probabilities-a new approach", Technological Forecasting and Social Change, 1980, 16, pages 343-349.

5. R.K. Sarin, "A sequential approach to cross-impact analysis", Futures, 1978, 10, pages $53-62$.

6. R.B. Mitchell, J. Tydeman, and R. Curnow, "Scenario generation: limitations and developments in cross-impact analysis", Futures, 1977, 9, pages 205215.

7. R.L. Keeney and H. Raiffa, Decisions with Multiple Objectives (John Wiley, 1976).

8. C.W. Kirkwood and S.M. Pollock, Methodology for Characterizing Potential Adtersaries of Nuclear Material Safeguards Systems (San Francisco, WoodwardCilyde (onsultants, November 1978).

9. S. Lichtenstein and B. Fischhoff, "Do those who know more also know more about how much they know?" Organizational Behaviour and Human Performance, 1977, 20, pages 159-183; A. Tversky and D. Kahneman, "Judgment under uncertainty: heuristics and biases", Science, 1974, 185, pages 1124-1131. 